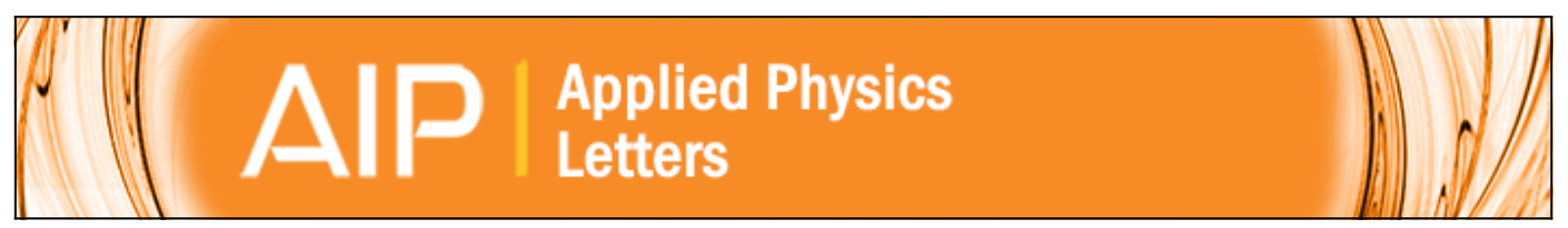

Pumping of water through carbon nanotubes by rotating electric field and rotating magnetic field

Xiao-Peng Li, Gao-Pan Kong, Xing Zhang, and Guo-Wei He

Citation: Applied Physics Letters 103, 143117 (2013); doi: 10.1063/1.4824441

View online: http://dx.doi.org/10.1063/1.4824441

View Table of Contents: http://scitation.aip.org/content/aip/journal/apl/103/14?ver=pdfcov

Published by the AIP Publishing 


\title{
Pumping of water through carbon nanotubes by rotating electric field and rotating magnetic field
}

\author{
Xiao-Peng Li, ${ }^{\text {a) }}$ Gao-Pan Kong, Xing Zhang, and Guo-Wei He ${ }^{\text {b) }}$ \\ LNM, Institute of Mechanics, Chinese Academy of Sciences, Beijing 100190, China
}

(Received 23 May 2013; accepted 20 September 2013; published online 3 October 2013)

\begin{abstract}
Using molecular dynamics simulations, we demonstrate pumping of water through a carbon nanotube by applying the combination of a rotating electric field and a rotating magnetic field. The driving force is a Lorentz force generated from the motion of charges in the magnetic field, and the motion is caused by the rotation of the electric field. We find that there exits a linear relationship between the average pumping velocity $v$ and magnetic field strength $B$, which can be used to control the flux of the continuous unidirectional water flow. This approach is expected to be used in liquid circulation without a pressure gradient. (C) 2013 AIP Publishing LLC. [http://dx.doi.org/10.1063/1.4824441]
\end{abstract}

Fluid transport in nanochannels is of great importance in many applications, including fluid filtration, biomolecule separation, energy conversion, thermal management, and drug delivery. ${ }^{1-7}$ The most widely used method to drive fluid through nanochannels is pressure difference..$^{8-18}$ In addition, various methods based on temperature gradients, ${ }^{19-21}$ mechanical vibration, ${ }^{22}$ surface energy gradient, $^{23}$ surface Rayleigh traveling waves, ${ }^{24}$ and pre-twisted wall ${ }^{25}$ were proposed to generate flow in the channels. Electro-osmosis methods, which yield unidirectional flows of water molecules by charges or external electric fields, are recognized as very promising approaches because they can be readily used to realize controllable flows in nanochannels. ${ }^{26-29}$ It was found that periodic vibration of a charge can be used to pump water through a carbon nanotube (CNT). ${ }^{28}$ However, static charges are not able to drive a continuous flow, although they can speed up the flow of polar liquids. ${ }^{30,31}$ Bonthuis et al. found that a rotating electric field in combination with the asymmetric slip boundary conditions can successfully generate a continuous flow in a planar or circular nanochannel. ${ }^{32,33}$ Rinne et al. demonstrated that water can be driven through a CNT by time-dependent electric fields generated by electrodes with oscillating charges. ${ }^{34}$ However, whether or not a static electric field can drive a continuous flow is still controversial. For instance, Joseph and Aluru ${ }^{35}$ and Wang et al. ${ }^{27}$ successfully drove water molecules by applying an uniform ${ }^{36}$ and a linear gradient ${ }^{27}$ static electric field along a CNT axis, respectively. But Bonthuis et al. argued that this static field pumping is a computer artifact, ${ }^{32,33,37}$ and they believed that static electric fields cannot induce electroneutral polar fluid flow in nanochannels.

In the present paper, we propose an approach to continuously pump water through CNTs based on molecular dynamics (MD) simulations. This is achieved by combining a rotating electric field with a rotating magnetic field. The driving force of water flow is a Lorentz force generated from the motion of charges in the magnetic field. Our method, in which no any asymmetric slip boundary condition is needed,

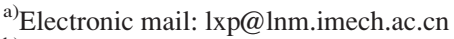

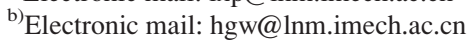

is different from pumping approach of Bonthuis et al. with rotating electric field. ${ }^{32,33}$

MD simulation is broadly used to study the transport properties of water in CNTs. ${ }^{5,6,31,38} \mathrm{We}$ will use the MD simulation to demonstrate that a rotating electric field and a rotating magnetic field can drive a continuous flow of water dipoles. Our simulations are performed using the LAMMPS molecular dynamics package ${ }^{39}$ with a time step of 1 fs. As shown in Fig. 1, an armchair $(6,6)$ CNT with $50.349 \AA$ in length and $8.125 \AA$ in diameter is constructed, and two reservoirs are added to the two tube ends. As movable pistons, two rigid planes are fixed in the $x$ - and $y$-direction and unfixed in the $z$-direction. The water molecules are modeled using the extended single point charge (SPC/E) model, ${ }^{40,41}$ and the whole system contains 6008 water molecules. The Lennard-Jones (L-J) potential is used to model water molecules in CNT when electric field is applied, as the same as what were done in Refs. 29, 32, 33, and 35. Therefore, the carbon atoms of CNT are modeled as uncharged L-J particles with $\sigma_{C C}=3.4 \AA$ and $\varepsilon_{C C}=0.359824 \mathrm{~kJ} / \mathrm{mol}^{42}$ Carboncarbon bond lengths of $1.418 \AA$ and bond angles of $120^{\circ}$ are maintained by harmonic potentials. The carbon-water L-J parameters are $\sigma_{C O}=3.19 \AA$ and $\varepsilon_{C O}=0.392 \mathrm{~kJ} / \mathrm{mol}^{43}$ In the simulations, a Chemistry at HARvard Macromolecular Mechanics (CHARMM) force field ${ }^{44,45}$ is used to calculate the L-J potentials and the electrostatic interactions with an additional switching function that ramps the energy and force smoothly to zero between an inner $(8 \AA)$ and outer $(10 \AA)$ cutoff distance. Periodic boundary conditions are applied in all directions. All simulations are performed in the canonical ensemble. A Nose-Hoover thermostat with a damping time of 0.1 ps is used to maintain the temperature of the water at $300 \mathrm{~K}$. The size of the simulation box is $5.1579 \times 5.105 \times 19 \mathrm{~nm}^{3}$. Since the water flow is not sensitive to the flexibility of CNT in the present simulations, we keep the carbon atoms fixed for the sake of simplicity, in the same way as those in Refs. 10, 15,17 , and 18 .

Water molecules enter the CNT from the reservoirs and form a single-file chain, ${ }^{15,42}$ as presented in Fig. 1. Two sinusoidal alternating electric fields are applied in the $x$ - and $y$-directions as follows: 


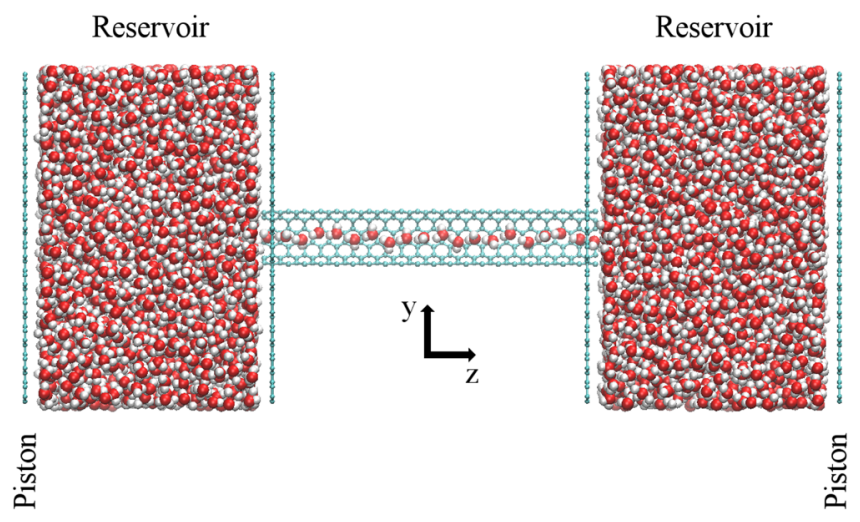

FIG. 1. Snapshot of the simulation system. Two reservoirs are connected by a CNT. The red and white balls represent the oxygen and hydrogen atoms in water molecules, respectively.

$$
\begin{gathered}
E_{x}=E \sin \left(2 \pi \times \frac{t}{T}\right), \\
E_{y}=E \sin \left[2 \pi \times\left(\frac{t}{T}-\frac{1}{4}\right)\right] .
\end{gathered}
$$

The joint effect of the two electric fields is an electric field which rotates around the $z$-direction, with strength $E$ and rotation period $T$ (the value of which will be given later). Furthermore, a rotating magnetic field is applied to the system, which is given by

$$
\begin{gathered}
B_{x}=-B \sin \left(2 \pi \times \frac{t}{T}\right), \\
B_{y}=-B \sin \left[2 \pi \times\left(\frac{t}{T}-\frac{1}{4}\right)\right] .
\end{gathered}
$$

That is to say, a magnetic field with strength $B$ is rotating together with the above electric field in the same period $T$, as sketched in Fig. 2(a). The direction of the magnetic field is always parallel to the direction of the electric field. Then, we find that a continuous water flow can be pumped by the rotating electric field and magnetic field (see the movie ${ }^{46}$ ).

As we know, if a particle of charge $q$ moves with velocity $\mathbf{v}_{\boldsymbol{q}}$ in the presence of a magnetic field $\mathbf{B}$, then the Lorentz force exerted on it is given by

$$
\mathbf{F}=q \mathbf{v}_{\mathbf{q}} \times \mathbf{B}
$$

A water molecule can be regarded as a dipole with a positive charge at one end and a negative charge at the other end.
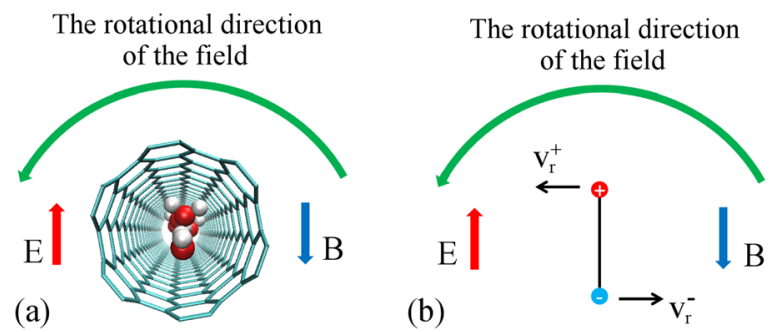

FIG. 2. (a) Sketch of the electric field $E$, magnetic field $B$, and the rotational direction of the field. (b) Sketch of a water dipole and the linear velocity of the charge $v_{r}^{+}$and $v_{r}^{-}$.
When a rotating electric field is applied, the water dipole will rotate with it and be always parallel to it. Therefore, the two ends of the water dipole move with linear velocities $v_{r}^{+}$ and $v_{r}^{-}$(perpendicular to the direction of the magnetic field), respectively. Since the rotations of the magnetic field and the electric field are synchronized, the water dipole vector and the magnetic field are always parallelled, as sketched in Fig. 2(b). Hence, the linear velocities of positive and negative charge on the water dipole are always perpendicular to the direction of magnetic field. As a result, the Lorentz force has a $z$-directional component $F_{z}$, in the axial direction of CNT, as sketched in Fig. 3. This component drives the water dipoles to move in the CNT.

MD simulations with different $B, E$, and $T$ are performed. For each case, the time for the simulation is $300 \mathrm{~ns}$. The simulation system is sufficiently relaxed during the beginning $30 \mathrm{~ns}$, and the last $270 \mathrm{~ns}$ of the simulation are collected for analysis.

Fig. 4(a) plots the dependence of average pumping velocity $v$ on the magnetic field $B$ at a given electric field of $E=0.5 \mathrm{~V} / \AA$ and $T=10 \mathrm{ps}$. Here, the average pumping velocity $v$ is defined as the average velocity of all water molecules in the CNT. Obviously, a non-zero $v$ implies a substantial continuous flow. We can find from Fig. 4(a) that the $v$ is much larger than zero for $B$ located at $[10,100]$. Therefore, the combination of a rotating electric field and a rotating magnetic field can generate a continuous flow for certain values of $B$ and $E$. It is also found that $v$ increases linearly as $B$ increases (the red solid line is the linear fit). Rinne et al. ${ }^{34}$ assumed that there exits a linear relationship between the external force and the velocity of water molecules in CNT. That is,

$$
v=\frac{N \bar{F}_{z}}{\gamma L},
$$

where $N$ is the number of water molecules inside the CNT, $L$ is the length of the CNT, $\bar{F}_{z}$ is the average of $F_{z}$, and $\gamma$ is the friction coefficient per unit length. Furthermore, according to Eq. (5), $F_{z}$ is proportional to the magnetic field strength $B$. Thus, it implies that a linear relationship exists between $v$ and $B$. This is consistent with our numerical results.

Fig. 4(b) presents the dependence of $v$ on the electric field strength $E$ at a given magnetic field of $B=50$ Tesla and $T=10$ ps. Similar results as those in Fig. 4(a) are observed:

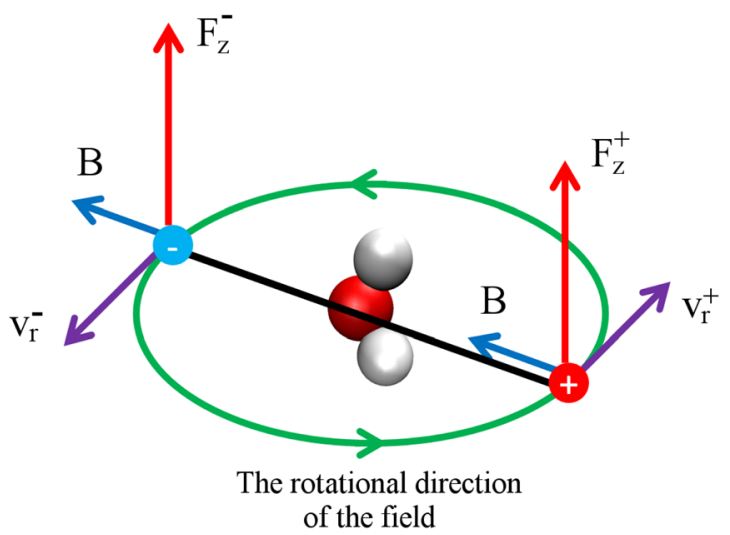

FIG. 3. Sketch of the Lorentz force $F_{z}$ on a water dipole. 

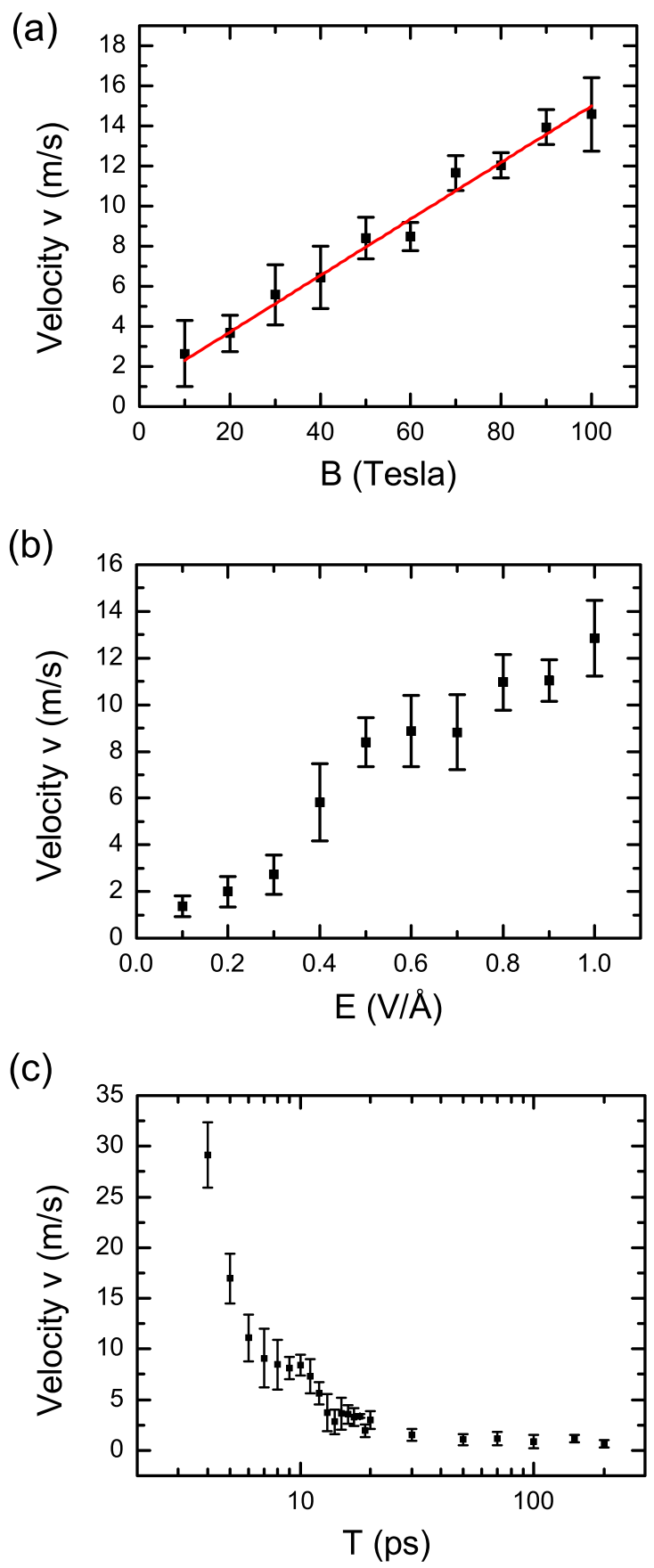

FIG. 4. The average pumping velocity $v$ (data points obtained from MD simulations). (a) $v$ as a function of the magnetic field strength $B(E=0.5 \mathrm{~V} / \AA$, $T=10 \mathrm{ps})$. (b) $v$ as a function of the electric field strength $E(B=50$ Tesla, $T=10 \mathrm{ps})$. (c) $v$ as a function of the field rotating period $T(E=0.5 \mathrm{~V} / \AA$, $B=50$ Tesla).

$v$ is significantly larger than zero and increases as $E$ increases. The observations again confirm that the combination of a rotating electric field and a rotating magnetic field can generate a continuous flow.

Fig. 4(c) investigates the dependence of $v$ on the period $T$. We can find that the $v$ decreases to almost zero as $T$ increases to infinity. This implies that a combination of static electric field and static magnetic field could not generate a continuous flow. Therefore, rotations of electric field and magnetic field are necessary for continuous flow.

In addition, our simulations show that the water dipoles rotate with the external rotating electric field when $T$ is greater than $3 \mathrm{ps}$, but cannot catch up with the rotation of the external electric field when $T$ is less than 3 ps. Details about this result are presented in supplementary material. ${ }^{46}$ Because there is always a lag between the rotation of water dipoles and the rotation of electric field, the water dipoles lose their response to the electric field when the field frequency is higher than a critical value (i.e., the rotation period $T$ of the field is less than a critical value). A molecule rotates about 1 radian per ps in bulk fluid, thus the critical value of $T$ in bulk is about $6 \mathrm{ps}$. It is found from our simulations that the critical value for water molecules inside CNTs is about 3 ps, half of it in bulk. The reason is that the water molecules confined inside the CNT have fewer neighbors than those in bulk; thus, their response to electric fields is faster than those in bulk.

To study the necessity of using rotating magnetic field in fluids pumping, we further investigate the individual effect of rotating electric field on the single-file water molecules inside a CNT. First, when a static external electric field, perpendicular to the axial direction of the CNT, is applied on the system, the water dipoles are observed in alignment with the direction of this electric field due to dipolar polarization. Figs. 5(a) and 5(b) show that the form of single-file water chain inside the CNT is arranged as follows: random arrangement, tilted staggered arrangement, and vertical staggered arrangement when the electric field strength $E$ equals $0,0.5$, and $1 \mathrm{~V} / \AA$, respectively. Fig. 6 plots the distribution of the water dipole deviation angle $\theta$ when $E$ varies from 0.3 to $1.5 \mathrm{~V} / \AA$, where $\theta$ is defined as the instantaneous angle between the water dipole vector and the direction of applied electric field. Every MD simulation is performed for $10 \mathrm{~ns}$ and outputs instantaneous angle $\theta$ per $10 \mathrm{ps}$. We calculated the distribution based on all of the output $\theta$. From the Fig. 6 , as $E$ increases, the peak position of the distribution moves toward $0^{\circ}\left(0^{\circ}\right.$ denotes the water dipole vector pointing towards the direction of the electric field $E$ ) and the peak value also increases, which means that the dipolar polarization of water dipoles become stronger as $E$ increases. When the electric (a) Side view

$\mathrm{E}=0 \mathrm{~V} / \AA$, random arrangement

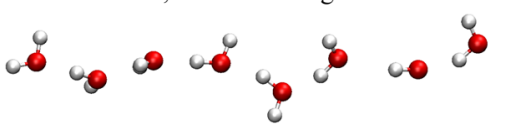

$\mathrm{E}=0.3 \mathrm{~V} / \AA$, tilted staggered arrangement

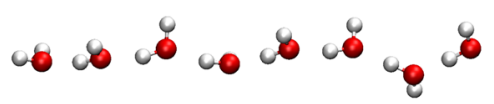

$\mathrm{E}=0.5 \mathrm{~V} / \AA$, tilted staggered arrangement

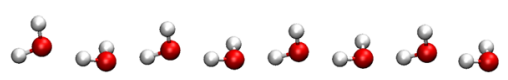

$\mathrm{E}=1 \mathrm{~V} / \AA$, vertical staggered arrangement

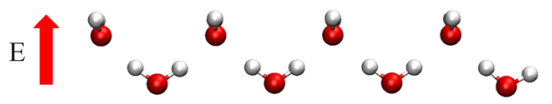

(b) Top view
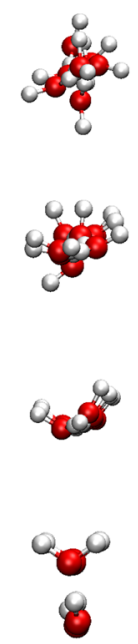

FIG. 5. The form of single-file water chain inside the CNT when $E$ is $0 \sim 1 \mathrm{~V} / \AA$. The electric field direction is upward. Carbon nanotubes are not displayed for clarity. (a) Side view of the simulation snapshot. (b) Top view of the simulation snapshot. 


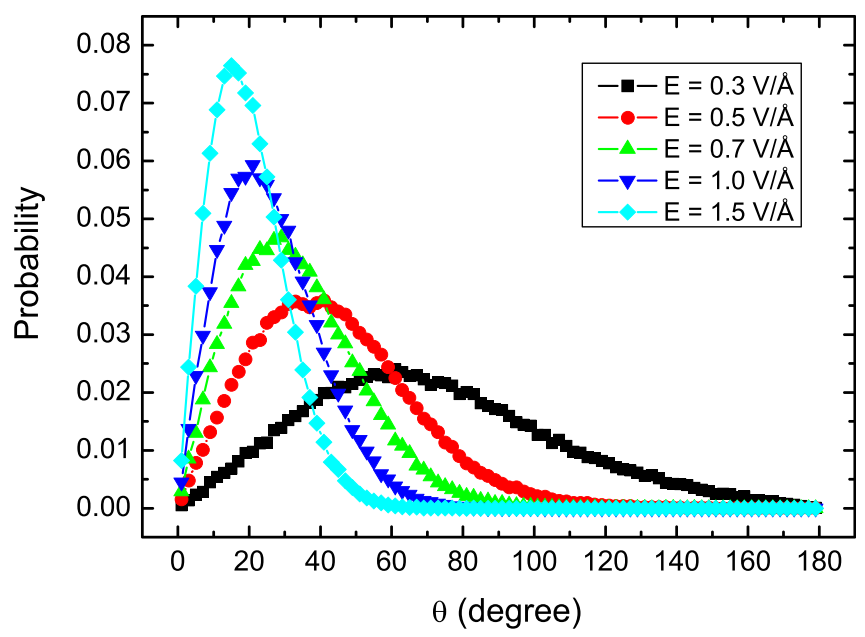

FIG. 6. The distribution of the water dipole deviation angle $\theta .0^{\circ}$ denotes the water dipole vector pointing towards the direction of the electric field $E$.

field rotates, the water dipoles are found rotating with it, keeping the form of single-file water chain unchanged, and not moving along the CNT. Overall, water molecules cannot be driven through CNTs by only using a rotating electric field.

To summarize, we propose to use the combination of a rotating electric fields and a rotating magnetic field to drive a continuous water flow through nanotube. Its mechanism is explained as follows: A rotating electric field forces water dipoles to rotate in the same way as the rotating electric field does. As a result, the velocity of each electric charge on the water dipole is kept to be perpendicular to the direction of the electric field. Meanwhile, a rotating magnetic field is applied in the same direction as the electric field. Therefore, a Lorentz Force is generated unidirectionally along the axis of CNT, which drives a continuous flow of water dipoles. This pumping approach can take place in a non-contact process and is expected to be applied in liquid circulation without osmotic pressure and hydrostatic pressure gradient.

We thank Dr. Xiao-Di Wang for helpful discussions. Computational resources were provided by the National Supercomputer Center in Tianjin. This work was supported by the National Natural Science Foundation of China (Grant Nos. 11232011 and 11021262) and the National Basic Research Program of China (Grant No. 2013CB834100) (Nonlinear science).

${ }^{1}$ T. M. Squires and S. R. Quake, Rev. Mod. Phys. 77, 977 (2005).

${ }^{2}$ J. K. Holt, Science 312, 1034 (2006).

${ }^{3}$ G. M. Whitesides, Nature 442, 368 (2006).

${ }^{4}$ M. Whitby and N. Quirke, Nat. Nanotechnol. 2, 87 (2007).
${ }^{5}$ J. C. Rasaiah, S. Garde, and G. Hummer, Annu. Rev. Phys. Chem. 59, 713 (2008).

${ }^{6}$ Y. Li, J. Xu, and D. Li, Microfluid. Nanofluid. 9, 1011 (2010).

${ }^{7}$ L. Bocquet and E. Charlaix, Chem. Soc. Rev. 39, 1073 (2010).

${ }^{8}$ F. Zhu, E. Tajkhorshid, and K. Schulten, Biophys. J. 83, 154 (2002).

${ }^{9}$ A. Kalra, S. Garde, and G. Hummer, Proc. Natl. Acad. Sci. U.S.A. 100, 10175 (2003).

${ }^{10}$ S. Joseph and N. R. Aluru, Nano Lett. 8, 452 (2008).

${ }^{11}$ M. E. Suk, A. V. Raghunathan, and N. R. Aluru, Appl. Phys. Lett. 92, 133120 (2008).

${ }^{12}$ L. Liu, Y. Qiao, and X. Chen, Appl. Phys. Lett. 92, 101927 (2008).

${ }^{13}$ J. A. Thomas and A. J. H. McGaughey, Nano Lett. 8, 2788 (2008).

${ }^{14}$ M. E. Suk and N. R. Aluru, Phys. Chem. Chem. Phys. 11, 8614 (2009).

${ }^{15}$ J. A. Thomas and A. J. H. McGaughey, Phys. Rev. Lett. 102, 184502 (2009).

${ }^{16}$ M. E. Suk and N. R. Aluru, J. Phys. Chem. Lett. 1, 1590 (2010).

${ }^{17}$ K. Falk, F. Sedlmeier, L. Joly, R. R. Netz, and L. Bocquet, Nano Lett. 10, 4067 (2010)

${ }^{18}$ B. Xu, Y. Qiao, Q. Zhou, and X. Chen, Langmuir 27, 6349 (2011).

${ }^{19}$ M. J. Longhurst and N. Quirke, Nano Lett. 7, 3324 (2007).

${ }^{20}$ H. A. Zambrano, J. H. Walther, P. Koumoutsakos, and I. F. Sbalzarini, Nano lett. 9, 66 (2009).

${ }^{21}$ C. Liu and Z. Li, Phys. Rev. Lett. 105, 174501 (2010).

${ }^{22}$ H. Qiu, R. Shen, and W. Guo, Nano Res. 4, 284 (2011).

${ }^{23}$ J. Kou, M. Mei, H. Lu, F. Wu, and J. Fan, Phys. Rev. E 85, 056301 (2012).

${ }^{24}$ Z. Insepov, D. Wolf, and A. Hassanein, Nano Lett. 6, 1893 (2006).

${ }^{25}$ W. H. Duan and Q. Wang, ACS Nano 4, 2338 (2010).

${ }^{26}$ J. Su and H. Guo, ACS Nano 5, 351 (2011).

${ }^{27}$ Y. Wang, Y. J. Zhao, and J. P. Huang, J. Phys. Chem. B 115, 13275 (2011).

${ }^{28}$ J. Kou, X. Zhou, H. Lu, Y. Xu, F. Wu, and J. Fan, Soft Mater. 8, 12111 (2012).

${ }^{29}$ X. Gong, J. Li, C. Guo, K. Xu, and H. Yang, Nanotechnology 24, 025502 (2013).

${ }^{30}$ J. Wong-ekkabut, M. S. Miettinen, C. Dias, and M. Karttunen, Nat. Nanotechnol. 5, 555 (2010).

${ }^{31}$ G. Zuo, R. Shen, S. Ma, and W. Guo, ACS Nano 4, 205 (2010).

${ }^{32}$ D. J. Bonthuis, D. Horinek, L. Bocquet, and R. R. Netz, Phys. Rev. Lett. 103, 144503 (2009).

${ }^{33}$ D. J. Bonthuis, D. Horinek, L. Bocquet, and R. R. Netz, Langmuir 26, 12614 (2010)

${ }^{34}$ K. F. Rinne, S. Gekle, D. J. Bonthuis, and R. R. Netz, Nano Lett. 12, 1780 (2012).

${ }^{35}$ S. Joseph and N. R. Aluru, Phys. Rev. Lett. 101, 64502 (2008).

${ }^{36}$ M. E. Suk and N. R. Aluru, Phys. Rev. Lett. 105, 209402 (2010).

${ }^{37}$ D. J. Bonthuis, K. Falk, C. N. Kaplan, D. Horinek, A. N. Berker, L. Bocquet, and R. R. Netz, Phys. Rev. Lett. 105, 209401 (2010).

${ }^{38}$ A. Alexiadis and S. Kassinos, Chem. Rev. 108, 5014 (2008).

${ }^{39}$ S. Plimpton, J. Comput. Phys. 117, 1 (1995).

${ }^{40}$ H. J. C. Berendsen, J. R. Grigera, and T. P. Straatsma, J. Phys. Chem. 91, 6269 (1987).

${ }^{41}$ D. van der Spoel, P. J. van Maaren, and H. J. C. Berendsen, J. Chem. Phys. 108, 10220 (1998).

${ }^{42}$ G. Hummer, J. C. Rasaiah, and J. P. Noworyta, Nature 414, 188 (2001).

${ }^{43}$ T. Werder and J. Walther, J. Phys. Chem. B 107, 1345 (2003).

${ }^{44}$ B. R. Brooks, R. E. Bruccoleri, B. D. Olafson, D. J. States, S. Swaminathan, and M. Karplus, J. Comput. Chem. 4, 187 (1983).

${ }^{45}$ A. D. MacKerell, D. Bashford, M. Bellott, R. L. Dunbrack, J. D. Evanseck, M. J. Field, S. Fischer, J. Gao, H. Guo, S. Ha et al., J. Phys. Chem. B 102, 3586 (1998).

${ }^{46}$ See supplementary material at http://dx.doi.org/10.1063/1.4824441 for a movie of water flow, and details about MD simulations to detect the critical value of the rotation period $T$. 